\title{
Intervertebral disc degeneration relates to biomechanical changes of spinal ligaments
}

\section{Journal Article}

Author(s):

Cornaz, Frédéric; Widmer, Jonas; Farshad-Amacker, Nadja A.; Spirig, José M.; Snedeker, Jess Gerrit (i); Farshad, Mazda

Publication date:

2021-08

Permanent link:

https://doi.org/10.3929/ethz-b-000499994

Rights / license:

Creative Commons Attribution 4.0 International

Originally published in:

The Spine Journal 21(8), https://doi.org/10.1016/j.spinee.2021.04.016 


\title{
The SPINE JOURNAL
}

The Spine Journal 21 (2021) 1399-1407

\section{Basic Science}

\section{Intervertebral disc degeneration relates to biomechanical changes of spinal ligaments}

\author{
Frédéric Cornaz, Dr Med ${ }^{\mathrm{a}, \mathrm{b}}$, Jonas Widmer, $\mathrm{PhD}^{\mathrm{a}, \mathrm{b}}$, \\ Nadja A. Farshad-Amacker, PD Dr Med ${ }^{\mathrm{c}}$, José Miguel Spirig, Dr Med ${ }^{\mathrm{a}}$, \\ Jess G. Snedeker, Prof Dr ${ }^{\mathrm{a}, \mathrm{b}}$, Mazda Farshad, Prof Dr Med MPH ${ }^{\mathrm{a}, *}$ \\ ${ }^{a}$ Department of Orthopaedics, Balgrist University Hospital, Zurich, Switzerland \\ ${ }^{\mathrm{b}}$ Institute for Biomechanics, ETH Zurich, Zurich, Switzerland \\ ${ }^{\mathrm{c}}$ Radiology, Balgrist University Hospital, Zurich, Switzerland \\ Received 7 March 2021; revised 2 April 2021; accepted 16 April 2021
}

Abstract

BACKGROUND CONTEXT: The ligamentum flavum (LF), the inter- and supraspinous ligament (ISL\&SSL) and the intertransverse ligament (ITL) are relevant spinal structures for segmental stability. The biomechanical effect of degeneration and aging on their biomechanical properties remains largely unknown.

PURPOSE: The aim of this study was to assess the material properties of the ITL, ISL\&SSL and LF and to correlate parameters of biomechanical function with LF-thickness, intervertebral disc (IVD) degeneration and age.

STUDY DESIGN: Biomechanical cadaveric study.

METHODS: MRI- and CT-scans of 50 human lumbar segments (Th12-L5) were used to assess the ISL (acc. to Keorochana), the grade of IVD degeneration (acc. to Pfirrmann) and to quantify LFthickness. The ITL, ISL\&SSL and LF were resected in the neutral position of the spinal segment with a specifically developed method to conserve initial strain. Ramp to failure testing was performed $(0.5 \mathrm{~mm} / \mathrm{s})$ to record initial tension, slack length, stiffness and ultimate strength. The relationship between the biomechanical characteristics and age and radiological parameters were analyzed. There are no study-specific conflicts of interest and no external funding was received for this study.

RESULTS: With aging, a significant reduction in initial tension $(\mathrm{r}=-0.5, \mathrm{p}<.01)$ and ultimate strength $(\mathrm{r}=-0.41, \mathrm{p}<.01)$ of the LF was observed, while the effect on LF-stiffness and the characteristics of the other ligaments was non-significant. IVD-degeneration was correlated with a significant reduction in stiffness $(\mathrm{r}=-0.47, \mathrm{p}=.001 ; \mathrm{r}=-0.36, \mathrm{p}=.01)$ and ultimate strength $(\mathrm{r}=-0.3, \mathrm{p}=.04$; $\mathrm{r}=-0.36, \mathrm{p}=.01$ ) of the LF and ISL\&SSL respectively and a significant reduction in initial tension $(\mathrm{r}=-0.4, \mathrm{p}<.01)$ of the LF. For the ITL, no significant correlation was observed. Comparing Pfirrman 2 to 5 , this reduction was $40 \%$ to $80 \%$ for stiffness $60 \%$ to $70 \%$ for ultimate strength and $88 \%$ for initial tension of the LF. ISL\&SSL-stiffness between Kerorochana grade A and D differed significantly ( $\mathrm{p}=.03$ ), while all other comparisons were non-significant ( $\mathrm{p}>.05)$. LF-thickness did not correlate with the biomechanical properties of the LF ( $p>.05)$.

CONCLUSIONS: Aging is primarily related to biomechanical changes to the LF. IVD-degeneration is related to a relevant reduction in stiffness and ultimate strength of the LF and ISL\&SSL, with a similar trend for the ITL. The ISL-specific Keorochana grading system provides only minimal biomechanical information and LF-thickness does not provide biomechanical information.

FDA device/drug status: Not applicable.

Author Disclosures: $\boldsymbol{F C}$ : Nothing to disclose. $\boldsymbol{J W}$ : Nothing to disclose. NAFA: Nothing to disclose. JMS: Nothing to disclose. JGS: Nothing to disclose. MF: Stock Ownership: Incremed (University start-up, member of the boards and stock owner, nonfinancial); Board of Directors: Incremed (University start-up, member of the boards and stock owner); Grants:
Medacta Spine Research support (A); Fellowship Support: Deputy Synthes Spine Fellowship support (A).

*Corresponding author: Balgrist University Hospital, Department of Orthopaedics, University of Zurich, Forchstrasse 340, CH - 8008 Zurich, Switzerland, Tel.: +41 44386 3004; Fax: +41 443863009.

E-mail address: mazda.farshad@balgrist.ch (M. Farshad). 
CLINICAL SIGNIFICANCE: Patient age and the degenerative state of the IVD can be used to evaluate the biomechanical characteristics of the dorsal spinal ligaments, which can be helpful in selecting the optimal surgical procedure (e.g. in decompression surgery) for a specific situation. (C) 2021 The Author(s). Published by Elsevier Inc. This is an open access article under the CC BY license (http://creativecommons.org/licenses/by/4.0/)

Keywords: $\quad$ Dorsal ligaments; Spine; Degeneration; Mechanical properties

\section{Introduction}

The spinal ligaments provide stability, while permitting motion. Mechanical properties [1-10], composition $[2,5,6]$, fiber orientation [5,7,11-13] as well as the biomechanical role in supporting the spine under load [13-16] have been investigated. The stress-strain curve of spinal ligaments shows a non-linear toe-region, a linear middle region and a plastic end region [2,3,17]. Similar to the intervertebral disc (IVD), the behavior is viscoelastic and therefore velocity dependent $[2,18]$. In the neutral position of the spine, the ligamentum flavum (LF) and the anterior and posterior longitudinal ligaments have been shown to be under initial tension $[4,5,8,19]$ (initial tension in the neutral position), while the intertransverse ligaments (ISL) and the supraspinous ligaments (SSL) were measured to be relaxed and engage only after a certain slack length $[2,4,9]$. The biomechanical role of spinal ligaments in healthy lumbar spines has been well characterized, however, only limited information on their biomechanical role in the degenerated spine is available.

While age-dependent reduction in stiffness and ultimate strength of the spinal ligaments have been described $[6,8,20]$, it remains controversial whether a cause-effect relationship exists. In patients with IVD-degeneration, far-reaching ultrastructural changes in the ISL\&SSL were observed [21]. These structural changes can be expected to result in alteration of the biomechanical integrity of the ligaments, however, this hypothesis has not been confirmed in a previous study [20]. Similarly, the initial tension of the LF has been measured to be reduced with aging [6], while the relation to segmental degeneration has not been evaluated.

Reduced contribution of the spinal ligaments to segmental stability could result in load-reallocation towards other anatomical structures such as the intervertebral disc or the facet joints and could lead to higher vulnerability to trauma. Knowledge about the biomechanical changes of the spinal ligaments during degeneration could also provide a new perspective on the processes responsible for the degenerative cascade and once fully understood, it could provide a helpful tool for the clinical evaluation of specific situations.

The aim of this study was to relate the mechanical properties of spinal ligaments (LF, ISL\&SSL and ITL) with age and clinically applicable radiological parameters: The Pfirrmann classification system [22], which is commonly used to evaluate the degenerative state of the IVD, the Keorochana grading system [23] for evaluation of the degenerative state of the ISL and LF-thickness, which has been proposed as a measure for the degenerative state of the LF [24-26]. We hypothesize that age and the radiological parameters are associated with the mechanical properties of the spinal ligaments.

\section{Material and methods}

\section{Specimen preparation and radiologic evaluation}

The specimens were resected from 50 spinal segments during the workflow of a displacement-controlled stepwise reduction study, during which the segments were loaded in the six major axes with physiological loading amplitudes. Further information is available in Widmer et al. [31,27]. The spinal segments originated from 22 fresh frozen cadavers stored at $-20^{\circ} \mathrm{C}$ (5 female and 17 males, age: $48-82$ years; Science Care, Phoenix, AZ, USA). Local authorities issued ethical approval (BASEC Nr. 2017-00874). After thawing to room-temperature, a 3T MRI scan (Magnetom Prisma, Siemens Medical Solutions, Erlangen, Germany) acquiring sagittal T2w turbo spin-echo dixon images, including water only (repetition time/ echo time $4000 \mathrm{~ms} / 86 \mathrm{~ms}$; Field of view (FOV) $100 \mathrm{~mm}$; matrix $512 \times 512$ pixels; echo numbers 1 ; slice thickness $4 \mathrm{~mm}$; flip angle 150; echo train length 17) and sagittal T1w turbo spin-echo (repetition time/echo time $650 \mathrm{~ms} / 10 \mathrm{~ms}$; FOV $100 \mathrm{~mm}$; matrix $512 \times 512$; echo numbers 1 ; slice thickness $4 \mathrm{~mm}$; flip angle 160), was performed in order to evaluate IVD-degeneration based on the Pfirrmann classification [22] and the Keorochana grading system [23].

Further, the specimens were scanned with a 128-slice Computed tomography (CT) scanner (SOMATOM Edge Plus, Siemens Medical Solutions, Erlangen, Germany) for the LF-thickness measurements [24]. The measurements of the LF were performed using multiplanar reformation CT images to ensure an exact axial axis in order to be able to measure the maximal thickness of each LF. Grading and measurements were conducted by experienced radiologists.

\section{Biomechanical testing}

During resection of the ligaments, the segments were secured in their neutral position, which was defined at the zero-crossing point in flexion-extension testing of the intact specimens as described by Widmer et. al. [27]. To allow for reproducible resection without changing initial strain, a specifically designed resection setup was used: The setup consists of two K-wire guides, which are positioned at a predefined distance with a hole pattern for Kirschner wires 

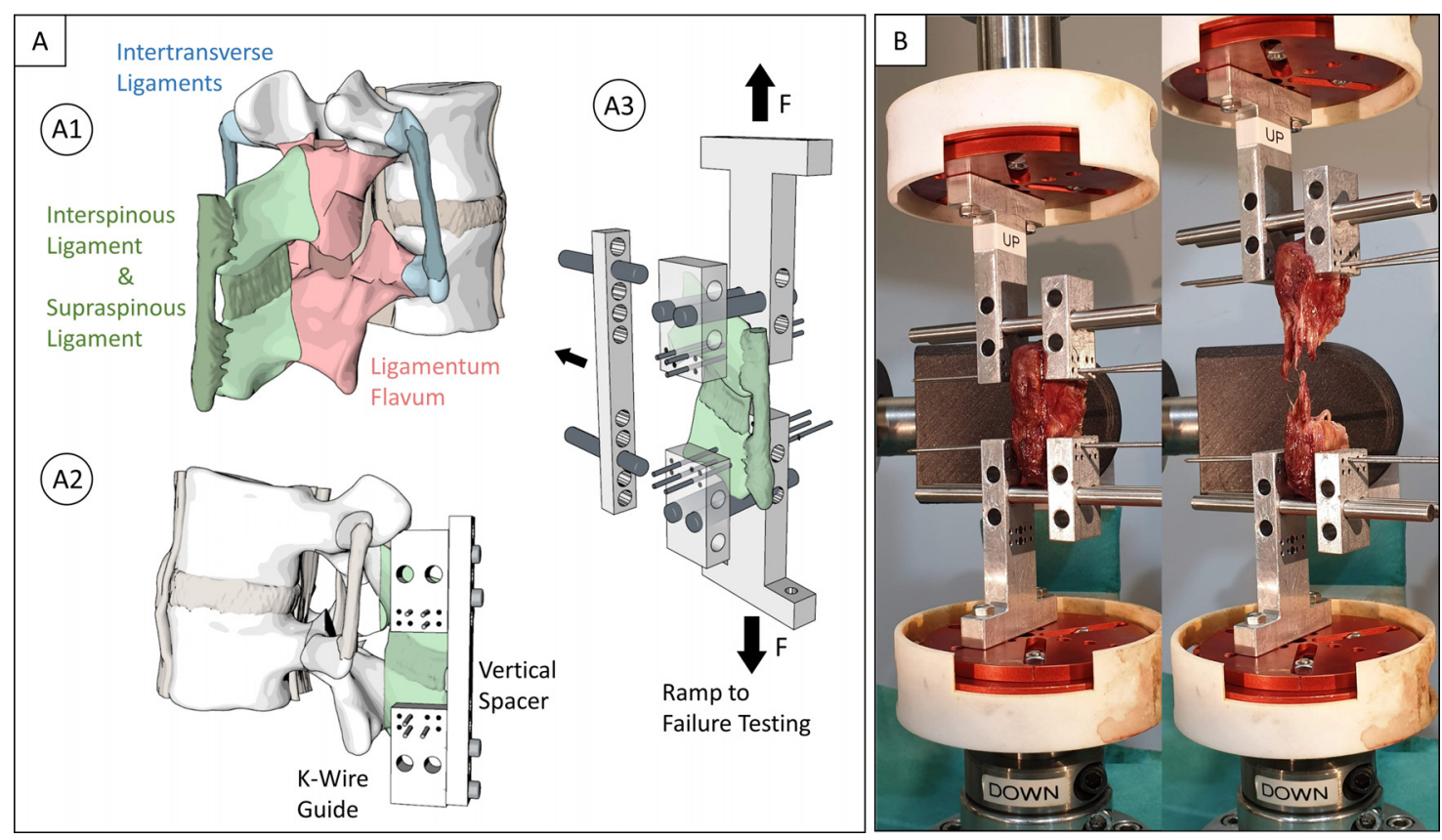

Fig. 1. Experimental procedure: (A1) The intertransverse ligament (blue), the interspinous and supraspinous ligament (green) and the ligamentum flavum (red) were analyzed in this study. (A2) Prior to resection, a transection guide was positioned, and Kirschner wires were drilled into the samples to conserve the original position. (A3) The transection guide was used to mount the sample into the tension-machine. (B) Ramp to failure testing was conducted (color version of figure is available online.)

(1.6 mm diameter, Dieter Marquardt Medizintechnik $\mathrm{GmbH}$, Germany) to be drilled into the transverse processes for ITL-resection, the spinous processes for ISL\&SSL-resection and the laminae for LF-resection (Fig. 1A). After Kirschner wire insertion, the bony attachment points of the ligaments were resected from the segment with an oscillating saw. The bone-tendon-bone specimens were mounted to the matching adapter system of the static testing machine (Fig. 1B) (Zwick/Roell Allroundline $10 \mathrm{kN}$ and testXpert III Software, ZwickRoell GmbH \& Co. KG, Germany). Prior to testing, the vertical spacers were removed. In cases with initial tension, the testing protocol contained an initial unloading phase. After reaching $0 \mathrm{~N}$, ramp to failure tensile testing was performed with a velocity of $0.5 \mathrm{~mm} / \mathrm{s}$ until a reduction in the maximal load of more than $20 \%$ was registered. Initial tension was defined as the measured force generated in the neutral position. The ISL\&SSL and LF were available for all 50 segments, while the ITL was available for 32 segments, due to absence in Th12/L1-segments and anatomical variability with very small transverse processes interfering with resection in some cases. The entire experiment was conducted within 12 hours from thawing to prevent tissue degradation. Specimen dehydration was prevented by frequent spraying with PBS solution. Initial hydration after thawing was not performed to prevent oversaturation.

\section{Data assessment and statistical evaluation}

Initial tension was measured directly after specimen transfer to the testing machine. In situations without initial tension, slack length was defined as the distance from the initial position to the position at which $1 \mathrm{~N}$ load was reached (Fig. 2). Stiffness was defined as the average of the slopes at $40 \%, 50 \%$ and $60 \%$ of the ultimate strength (Fig. 2), which corresponded to the linear region of the stress-strain curve. Ultimate strength was defined as the maximal point in the stress-strain curve (Fig. 2).

Non-parametric tests were used as normal distribution could not be assumed for all cases according to Shapiro-

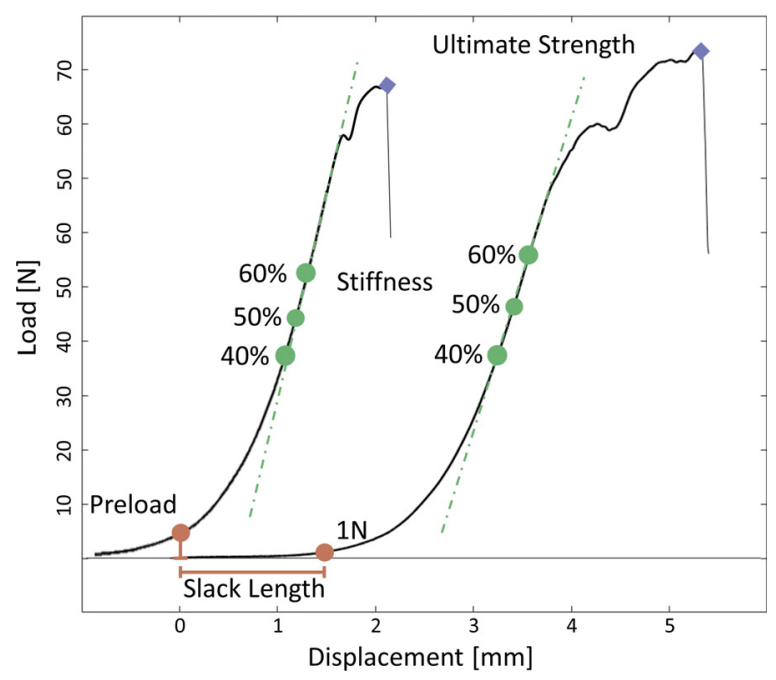

Fig. 2. Illustration of the data assessment for an example with initial tension (left curve) and an example with slack-length (right curve). Ultimate strength was defined as the maximal load measurement (blue square) and stiffness as the average of the slopes at $40 \%, 50 \%$ and $60 \%$ of the ultimate strength (green dots). Slack length was defined as the interval between $0 \mathrm{~N}$ to $1 \mathrm{~N}$ load (red line) and initial tension as the load in the initial position (red dot) (color version of figure is available online.) 

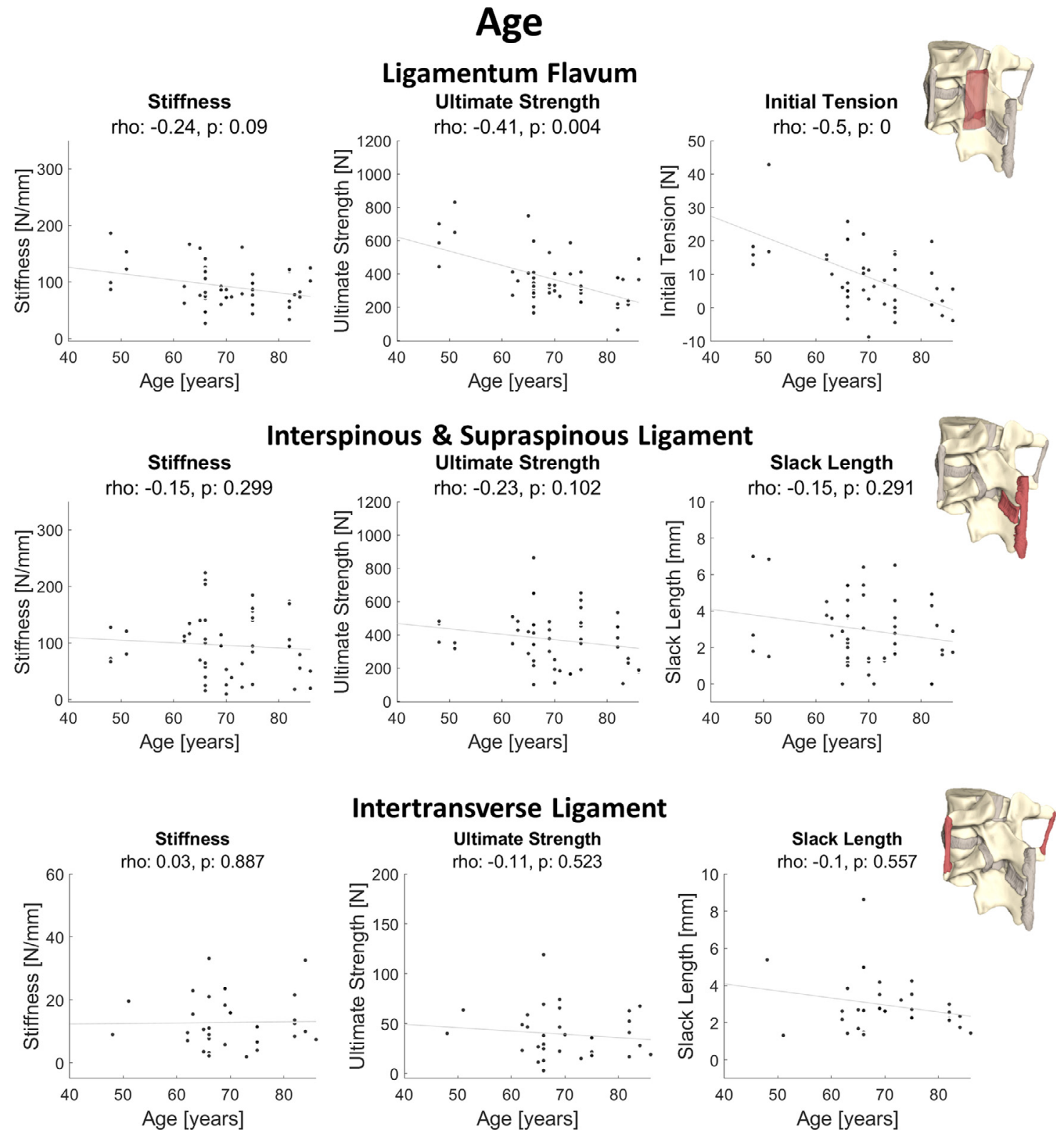

Fig. 3. Age dependent changes to the biomechanical characteristics of the ligamentum flavum, interspinous and supraspinous ligament and intertransverse ligament. (rho=spearman coefficient.)

Wilk tests. Spearman's rank tests were used to evaluate the monotone relation between the biomechanical characteristics of the ligaments and donor age, Pfirrmann grade and LF-thickness (Fig. 3,4,6). Due to the non-ordinal nature of the Keorochana grading system, Mann-Whitney U tests were used to compare grades (Fig. 5). Statistical significance was assumed with $\mathrm{p}<.05$.

\section{Results}

\section{Age-dependent changes}

For the LF, the association between age and stiffness did not reach statistical significance $(\rho=-0.24, \mathrm{p}=.09)$, while the relation between age and ultimate strength $(\rho=-0.41$, $\mathrm{p}=.004)$ and between age and initial tension $(\rho=-0.5$, $\mathrm{p}=.001)$ did. For the ISL\&SSL, no significant correlations between age and stiffness $(\rho=-0.15, \mathrm{p}=.30)$, between age and ultimate strength $(\rho=-0.23, \mathrm{p}=.10)$ and between age and slack length $(\rho=-0.15, \mathrm{p}=.29)$ were recorded. For the ITL, no significant correlation between age and stiffness ( $\rho=0.03, \mathrm{p}=.89)$, ultimate strength $(\rho=-0.11, \mathrm{p}=.52)$ and slack length $(\rho=-0.1, \mathrm{p}=.56)$ was observed (Fig. 3$)$.

\section{IVD-degeneration}

From 50 specimens, 9 were classified as Pfirrmann 2, 18 as Pfirmann 3, 20 as Pfirrmann 4 and 3 as Pfirrmann 5. The 


\section{Degeneration (Pfirrmann)}

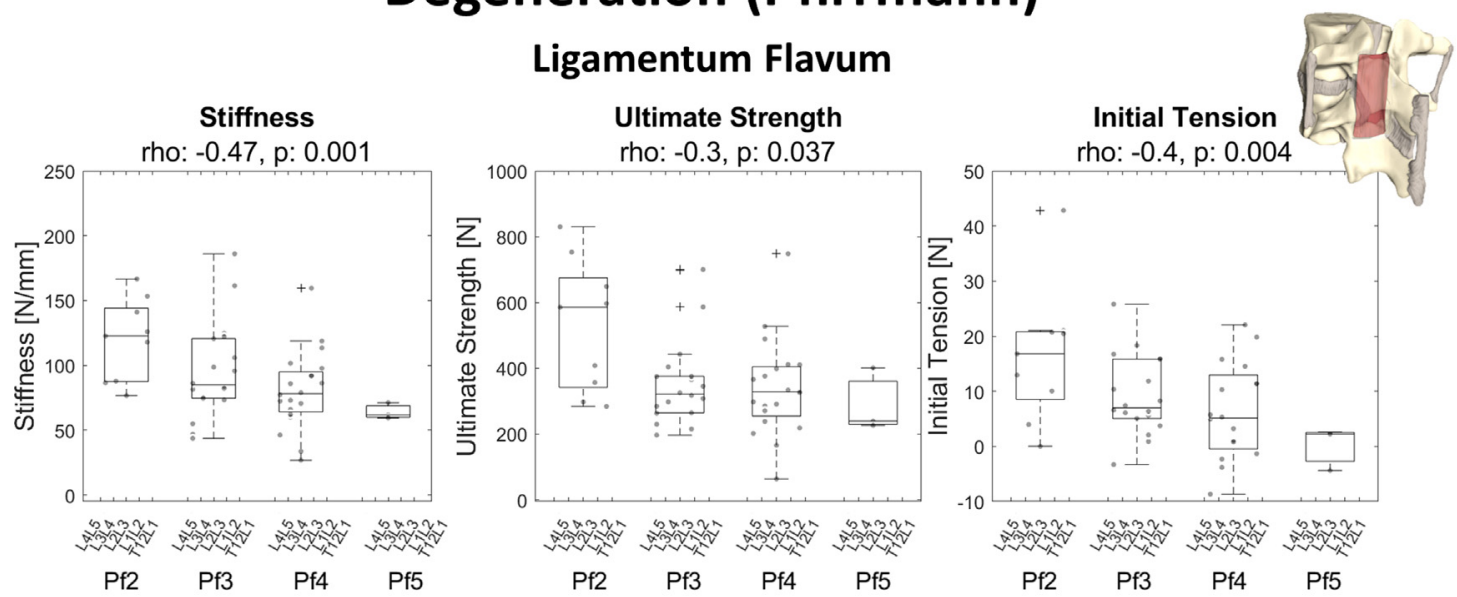

\section{Interspinous \& Supraspinous Ligament}
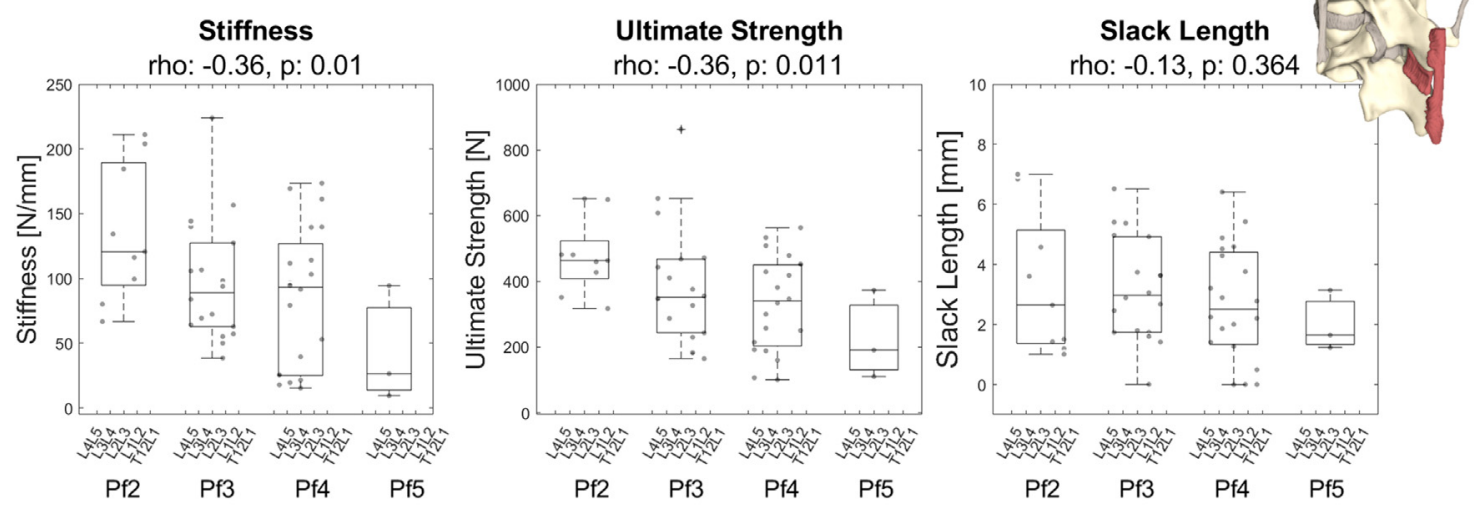

Intertransverse Ligament
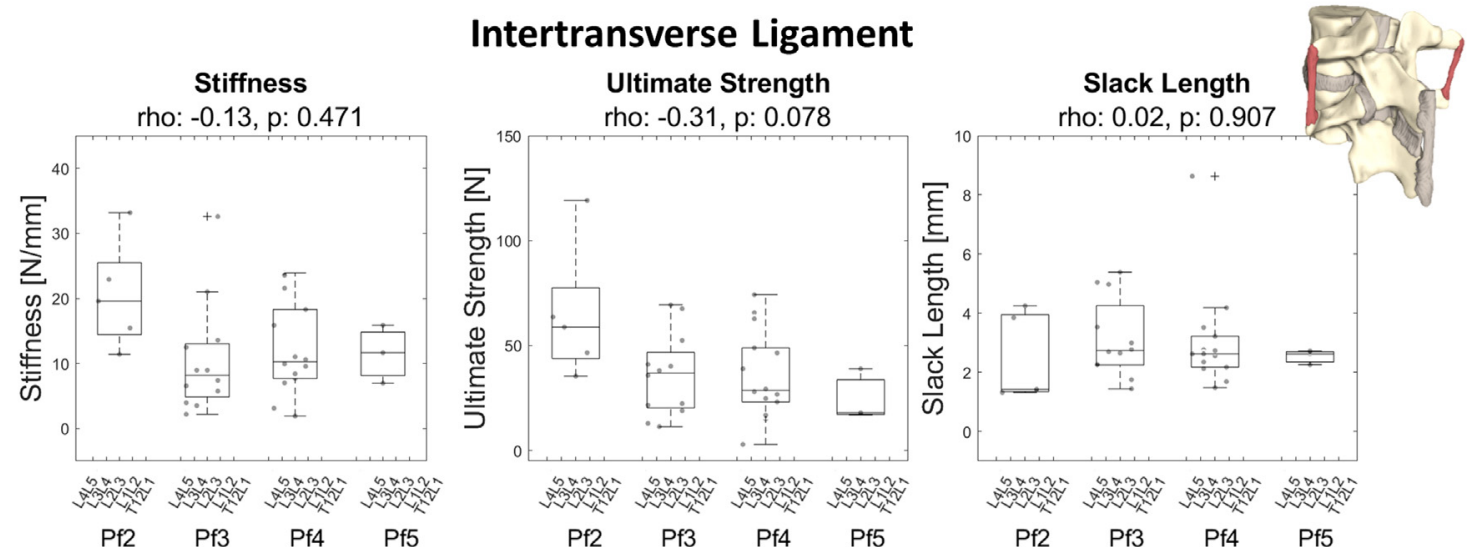

Fig. 4. Relation between intervertebral disc degeneration evaluated with the Pfirrmann classification system in relation to the biomechanical parameters of the ligamentum flavum, interspinous and supraspinous ligament and intertransverse ligament. (rho=spearman coefficient.)

distribution of the Pfirrmann classification on the spinal levels was analyzed (see Fig. A1 appendix, which illustrates the percentual level portion of each Pfirrmann classification group). For the LF, a significant negative correlation with stiffness $(\rho=-0.47, \mathrm{p}=.001)$, ultimate strength $(\rho=-0.3$, $\mathrm{p}=.037)$ and initial tension $(\rho=-0.4, \mathrm{p}=.004)$ was measured. Comparing Pfirrmann 2 to 5 , a $50 \%$ reduction in stiffness $(123 \mathrm{~N} / \mathrm{mm}-62 \mathrm{~N} / \mathrm{mm}), 60 \%$ in ultimate strength $(586$
$\mathrm{N}-239 \mathrm{~N})$ and $88 \%$ in initial tension $(17 \mathrm{~N}-2 \mathrm{~N})$ was recorded.

For the ISL\&SSL, a negative correlation for stiffness ( $\rho=-0.36, \mathrm{p}=.01)$ and ultimate strength $(\rho=-0.36, \mathrm{p}=.011)$ was observed, while the association to the slack length ( $\rho=-0.13, \mathrm{p}=.36$ ) was non-significant. Comparing Pfirrmann 2 to 5 , a $79 \%(121 \mathrm{~N} / \mathrm{mm}-26 \mathrm{~N} / \mathrm{mm})$ reduction in stiffness and $59 \%$ in ultimate strength $(464 \mathrm{~N}-191 \mathrm{~N})$ was recorded. 


\section{Keorochana}

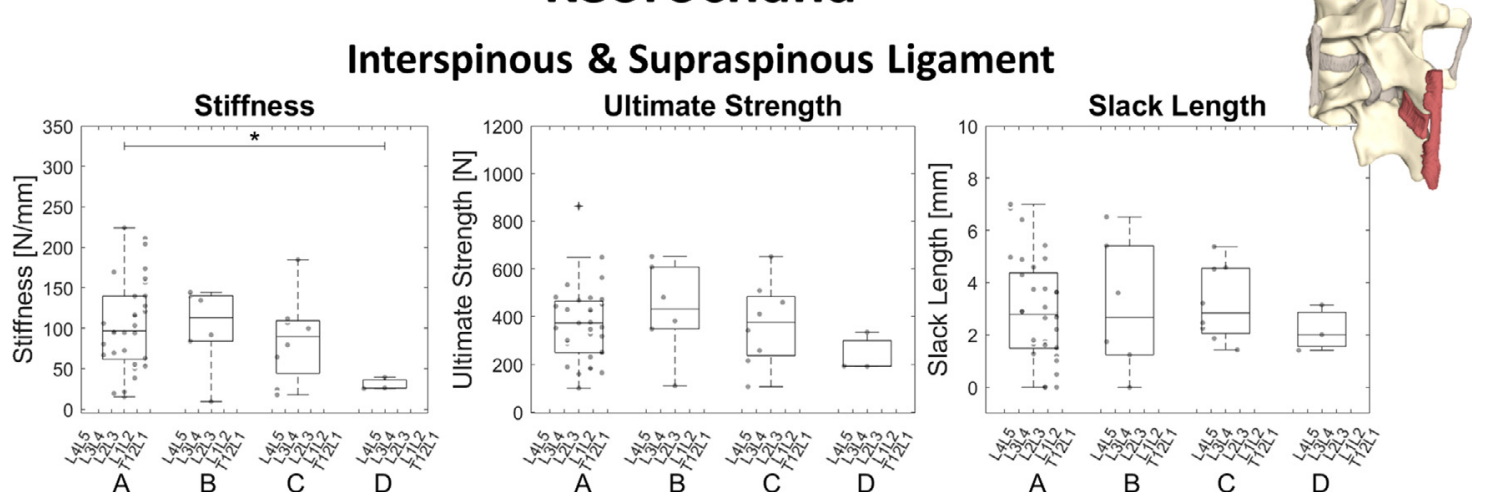

Fig. 5. Relation between Keorochana grading system and the interspinous and supraspinous ligament. Statistical significance $(\mathrm{p}<.05)$ is marked with an asterisk $(*)$.

For the ITL, the relationship to stiffness $(\rho=-0.13, \mathrm{p}=.47)$ and ultimate strength $(\rho=-0.31, \mathrm{p}=.078)$ did not reach significance. Comparing Pfirrmann 2 to 5 , a $40 \%$ reduction in stiffness $(20 \mathrm{~N} / \mathrm{mm}-12 \mathrm{~N} / \mathrm{mm})$ and $70 \%$ reduction in ultimate strength $(59 \mathrm{~N}-18 \mathrm{~N})$ was recorded. No significant correlation between Pfirrmann grade and slack length was recorded ( $\rho=0.02, \mathrm{p}=.91)$ (Fig. 4).

\section{ISL-degeneration (Keorochana grading system)}

According to the Keorochana grading system [23], 33 samples were classified as grade A, 6 samples as grade B, 8 samples as grade $\mathrm{C}$ and 3 samples as grade D. The stiffness-values of group A and D differed significantly (A: 97 $\mathrm{N} / \mathrm{mm}$; D: $26 \mathrm{~N} / \mathrm{mm}, \mathrm{p}=.03$ ). No significant differences between the other grades were observed (B: $113 \mathrm{~N} / \mathrm{mm}$; $\mathrm{C}$ : $100 \mathrm{~N} / \mathrm{mm})$. The differences in ultimate strength $(\mathrm{A}: 373 \mathrm{~N}$; B: $431 \mathrm{~N} ; \mathrm{C}: 411 \mathrm{~N}, \mathrm{D}: 191 \mathrm{~N})$ and slack length (A: 2.8 $\mathrm{mm}$; B: $2.6 \mathrm{~mm}$; C: $2.5 \mathrm{~mm}$, D: $2.0 \mathrm{~mm}$ ) were non-significant (p>.05) (Fig. 5).

\section{LF-thickness}

LF-thickness ranged from $1.6 \mathrm{~mm}$ to $4.7 \mathrm{~mm}$ (Mean: $3.3 \mathrm{~mm}$, Median $3.3 \mathrm{~mm}$ ) and did not correlate with stiffness $(\rho=0.21, \mathrm{p}=.16)$, ultimate strength $(\rho=0.23, \mathrm{p}=.105)$ or initial tension $(\rho=0.14, \mathrm{p}=.35)$ of the LF (Fig. 6).

\section{Discussion}

The relationship between the biomechanical properties of the spinal ligaments aging and radiological changes is not well understood. This information could help evaluate the role of the spinal ligaments in specific clinical situations. The aim of this study was to relate the mechanical properties of spinal ligaments (LF, ISL\&SSL and ITL) with age and promising, clinically applicable radiological parameters like the Pfirrmann classification system [22], the Keorochana grading system [23] and the LF-thickness.

Age-dependent changes to the biomechanical properties of spinal ligaments have been reported in the literature $[6,8,20]$. In our data, aging was primarily linked to a reduction in initial tension and ultimate strength of the LF, while

\section{Thickness}

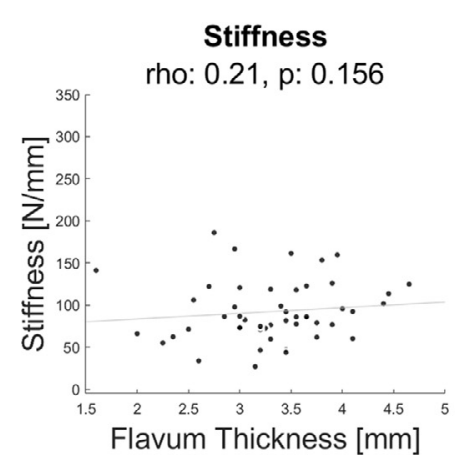

\section{Ligamentum Flavum}

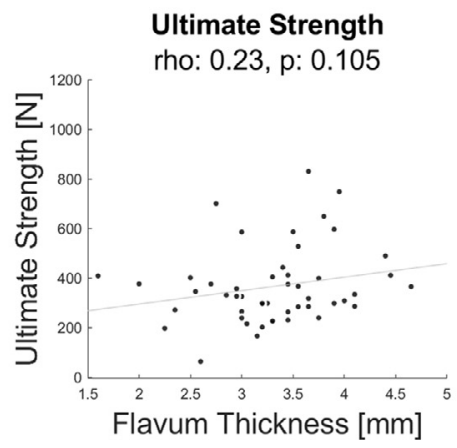

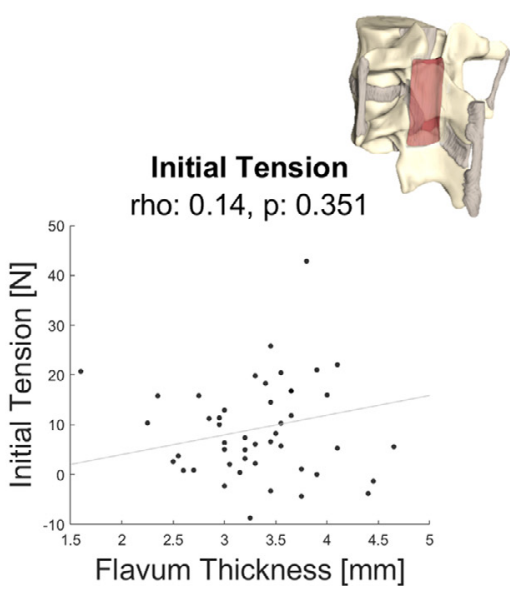

Fig. 6. Relation between ligamentum flavum thickness and the biomechanical characteristics of the ligamentum flavum. (rho=spearman coefficient.) 
the effect on LF-stiffness was non-significant. For the ITL and ISL\&SSL, the effect on ultimate strength could only be observed in a trend, while compared to LF, stiffness values were hardly changed. This difference between the LF and the ISL\&SSL and the ITL could be explained by the high elastin content in the LF, which is known to be reduced with aging [28]. Aging appears to have a more pronounced effect of the LF than on the other structures.

Comparing samples with mild IVD degeneration (Pfirrmann 2) with samples with severe IVD degeneration (Pfirrmann 5), a relevant reduction in stiffness $(40 \%-80 \%)$ and ultimate strength $(60 \%-70 \%)$ of the LF, the ITL and the ISL\&SSL and a notable reduction in initial tension of the LF (88\%) was observed. Similarly, significant correlations between IVD-degeneration and stiffness and ultimate strength of the LF and the ISL\&SSL were measured. Even though aging and degeneration are associated with each other and cannot be regarded independently, these results imply that ligament stiffness and potentially also ultimate strength could be more affected by degenerative changes to the IVD than by aging.

The stiffness-values of the ISL\&SSL-complex between group A and D of the Keorochana grading system [23], for which narrowing of the interspinous interval is the defining characteristic, differed significantly. Narrowing of the interspinous interval could be closely related to segmental degeneration and loss in disc height. While grade A, B and $\mathrm{C}$ differ in signal intensity and histological composition $[23,29]$, no significant differences in the biomechanical parameters were recorded. These results imply no strong biomechanical effect of the compositional changes which are responsible for the radiologic appearance of the ISL. However, detection of such a correlation could be prevented by the limited sample size, differences in radiologic appearances of cadaveric to living specimens and the measurement of the ISL\&SSL-complex instead of the isolated ISL. Even though the SSL provides more stiffness than the ISL [10], combined testing was favored due to the close relation between the two ligaments with the subsequent risk of damaging both ligaments during separation. Furthermore, since ISL\&SSL are usually either both resected or spared during spinal surgery, isolated analysis would not provide much additional information for the clinical practice.

Surprisingly, no significant correlation between LFthickness and the biomechanical characteristics of the LF was recorded. While small correlations could be detected with a larger sample size, LF-thickness does not appear to be a major factor in defining the ligament characteristics. We postulate two opposing effects, which could cancel each other out: While larger cross-sectional areas in healthy ligaments can be linked to higher stiffness, LF-thickening as a result of ligament fibrosis with a reduction in structural alignment has been discussed to weaken the ligament [24,26,30]. Further, radiological LF-thickness could also be a surrogate of a collapsing LF due to loss of disc height. In conclusion, radiologically measured LF-thickness does not provide information on the biomechanics of the LF.

In the clinical routine, knowledge about the effect of aging on the LF and the effect of disc degeneration on ligament stiffness and ultimate strength can help select the most adequate surgical procedure (e.g. decompression surgery) in a specific clinical situation. In the young, nondegenerated spine, the dorsal ligaments provide relevant biomechanical contribution, and in consequence, their integrity should be conserved by favoring unilateral microsurgical decompression over bilateral microsurgical decompression or laminectomy, if this approach is clinically justifiable. With aging and progressive IVD-degeneration, the contribution of the dorsal ligaments is markedly reduced, and the biomechanical effect of resection appears smaller and therefore less consequential. The reduction in biomechanical contribution with aging and spinal degeneration could further be an important factor in the degenerative cascade of the spine. A reduction in stiffness of the spinal ligaments could result in a reallocation of the load towards other structures such as the IVD and the facet joints [31], which could plausibly play an important role in the development of annulus fibrosus tears and facet joint osteoarthritis [31]. Spinal degeneration has also been observed to be related to erratic segmental movements [32,33] and to an unstable center of rotation [32], which can be explained by a reduced initial tension of the LF, which could compromise stability in the neutral zone of the segment.

The present findings need consideration of certain limitations. The specimens were obtained during a stepwise reduction study and the spinal segments were loaded prior to resection. However, with the applied method of the stepwise reduction study [27,31], only physiological loading conditions were applied and the potential effect on the later measurements should therefore be minimal. Testing was performed on cadaveric specimens at room temperature and while measures were taken to prevent alteration of the hydration state, potential effect on the measurements from these conditions cannot be excluded. Effects of postmortem storage might aggravate comparability of the mechanical properties from in-vivo conditions. However, it has previously been shown that biomechanical properties of ligaments and tendons after prolonged freezing storage are not different from those of fresh samples [34]. The neutral position at which resection was performed was chosen to be reproducible and to represent the physiological neutral position, however it was not possible to relate this position to in-vivo postures. A certain level-dependency of the spinal structures has been described $[3,10]$ and can also be seen in trends in our data. Pooling values from different spinal level may therefore interact with the results and their interpretation. However, with the relatively homogeneous level distribution for Pfirrmann Grade 2- 4 and only mid-lumbar segments for the Pfirrmann Grade 5 group (Appendix Fig. A1), this effect should be minimal. While the limited sample size could prevent the detection of small differences 
between the groups, we evaluate such potential differences as of minor importance for the segmental stability or the clinical practice.

\section{Conclusion}

While aging is primarily related to a reduction in initial tension and ultimate strength of the LF, IVD-degeneration is correlated with a relevant reduction in stiffness and ultimate strength of the LF and ISL\&SSL, with a similar trend for the ITL. Patient age and the degenerative state of the IVD can provide relevant information about the biomechanical characteristics of the dorsal spinal ligaments. The Keorochana grading system and LF-thickness provide only minimal biomechanical information.

\section{Acknowledgments}

Imaging was performed with support of the Swiss Center for Musculoskeletal Imaging, SCMI, Balgrist Campus AG, Zürich, with special acknowledgement to Natalie Hinterholzer and Prof. Daniel Nanz. The authors gratefully acknowledge the contribution of Dr. med. Julien Galley to the radiologic evaluation. No third-party funding was received for this work.

\section{Declarations of Competing Interests}

The authors declare that they have no known competing financial interests or personal relationships that could have appeared to influence the work reported in this paper.

\section{Supplementary materials}

Supplementary material associated with this article can be found in the online version at https://doi.org/10.1016/j. spinee.2021.04.016.

\section{References}

[1] Bradshaw RJ. Mechanical characterization of the interspinous ligament using anisotropic small punch testing. Mech Eng 2011: 2662. Available athttps://doi.org/http://scholarsarchive.byu.edu/ etd/2662/.

[2] Chazal J, Tanguy A, Bourges M, Gaurel G, Escande G, Guillot M, et al. Biomechanical properties of spinal ligaments and a histological study of the supraspinal ligament in traction. $\mathrm{J}$ Biomech 1985;18:167-76. https://doi.org/10.1016/0021-9290(85)90202-7.

[3] Pintar FA, Yoganandan N, Myers T, Elhagediab A, Sances A. Biomechanical properties of human lumbar spine ligaments. J Biomech 1992;25:1351-6. https://doi.org/10.1016/0021-9290(92)90290-H.

[4] Adams MA, Hutton WC, Stott JRR. The resistance to flexion of the lumbar intervertebral joint. Spine (Phila Pa 1976) 1980. https://doi. org/10.1097/00007632-198005000-00007.

[5] Hukins DW, Kirby MC, Sikoryn TA, Aspden RM, Cox AJ. Comparison of structure, mechanical properties, and functions of lumbar spinal ligaments. Spine (Phila Pa 1976) 1990;15:787-95. https://doi.org/ 10.1097/00007632-199008010-00010.

[6] Nachemson AL, Evans JH. Some mechanical properties of the third human lumbar interlaminar ligament (ligamentum flavum). J Biomech 2004;1:211-20. https://doi.org/10.1016/0021-9290(68)90006-7.
[7] Dickey JP, Bednar DA, Dumas GA. New insight into the mechanics of the lumbar interspinous ligament. Spine (Phila Pa 1976) 1996;21:2720 7. https://doi.org/10.1097/00007632-199612010-00004.

[8] Tkaczuk H. Tensile properties of human lumbar longitudinal ligaments. Acta Orthop Scand 1968;39:1-69. https://doi.org/10.3109/ ort.1968.39.suppl-115.01.

[9] Hindle RJ, Pearcy MJ, Cross A. Mechanical function of the human lumbar interspinous and supraspinous ligaments. J Biomed Eng 1990;12:340-4. https://doi.org/10.1016/0141-5425(90)90010-K.

[10] Myklebust JB, Pintar F, Yoganandan N, Cusick JF, Maiman D, Myers TJ, et al. Tensile strength of spinal ligaments. Spine (Phila Pa 1976) 1988;13:528-31. https://doi.org/10.1097/00007632-198805000-00016.

[11] Heylings DJ. Supraspinous and interspinous ligaments of the human lumbar spine. J Anat 1978;125:127-31

[12] Behrsin JF, Briggs CA. Ligaments of the lumbar spine: a review. Surg Radiol Anat 1988;10:211-9. https://doi.org/10.1007/BF02115239.

[13] Scapinelli R, Stecco C, Pozzuoli A, Porzionato A, Macchi V, De Caro R. The lumbar interspinous ligaments in humans: anatomical study and review of the literature. Cells Tissues Organs 2006;183:1-11. https://doi.org/10.1159/000094901.

[14] Hindle RJ, Pearcy MJ, Cross AT, Miller DHT. Three-dimensional kinematics of the human back. Clin Biomech 1990;5:218-28. https:// doi.org/10.1016/0268-0033(90)90005-Q.

[15] Harris RI, Macnab I. Structural changes in the lumbar intervertebral discs; their relationship to low back pain and sciatica. J Bone Joint Surg Br 1954;36-B:304-22. https://doi.org/10.1302/0301-620X.36B2.304.

[16] Pearcy MJ, Tibrewal SB. Lumbar intervertebral disc and ligament deformations measured in vivo. Clin Orthop Relat Res 1984:281-6 NO. 191. https://doi.org/10.1097/00003086-198412000-00038.

[17] Robertson D, Willardson R, Parajuli D, Cannon A, Bowdenn AE. The lumbar supraspinous ligament demonstrates increased material stiffness and strength on its ventral aspect. J Mech Behav Biomed Mater 2013;17:34-43. https://doi.org/10.1016/j.jmbbm.2012.07.009.

[18] Abrahams M. Mechanical behaviour of tendon. Med Biol Engng 1967;5:433-43. https://doi.org/10.1007/BF02479137.

[19] Neumann P, Keller TS, Ekström L, Perry L, Hansson TH, Spengler DM. Mechanical properties of the human lumbar anterior longitudinal ligament. J Biomech 1992;25:1185-94. https://doi.org/10.1016/ 0021-9290(92)90074-B.

[20] Iida T, Abumi K, Kotani Y, Kaneda K. Effects of aging and spinal degeneration on mechanical properties of lumbar supraspinous and interspinous ligaments. Spine J 2002;2:95-100. https://doi.org/ 10.1016/S1529-9430(02)00142-0.

[21] Yahia H, Drouin G, Maurais G, Garzon S, Rivard CH. Degeneration of the human lumbar spine ligaments: an ultrastructural study. Pathol Res Pract 1989;184:369-75. https://doi.org/10.1016/S0344-0338(89)80031-7.

[22] Pfirrmann CWA, Metzdorf A, Zanetti M, Hodler J, Boos N. Magnetic resonance classification of lumbar intervertebral disc degeneration. Spine (Phila Pa 1976) 2001;26:1873-8. https://doi.org/10.1097/ 00007632-200109010-00011.

[23] Keorochana G, Taghavi CE, Tzeng ST, Morishita Y, Yoo JH, Lee $\mathrm{KB}$, et al. Magnetic resonance imaging grading of interspinous ligament degeneration of the lumbar spine and its relation to aging, spinal degeneration, and segmental motion: clinical article. J Neurosurg Spine 2010;13:494-9. https://doi.org/10.3171/ 2010.4.SPINE09515.

[24] Sakamaki T, Sairyo K, Sakai T, Tamura T, Okada Y, Mikami H. Measurements of ligamentum flavum thickening at lumbar spine using MRI. Arch Orthop Trauma Surg 2009;129:1415-9. https://doi. org/10.1007/s00402-009-0849-1.

[25] Altun I, Yüksel KZ. Histopathological analysis of ligamentum flavum in lumbar spinal stenosis and disc herniation. Asian Spine J 2017;11:71-4. https://doi.org/10.4184/asj.2017.11.1.71.

[26] Chokshi FH, Quencer RM, Smoker WRK. The "thickened" ligamentum flavum: Is it buckling or enlargement? Am J Neuroradiol 2010;31:1813-6. https://doi.org/10.3174/ajnr.A2241. 
[27] Widmer J, Cornaz F, Scheibler G, Spirig JM, Snedeker JG, Farshad M. Biomechanical contribution of spinal structures to stability of the lumbar spine - novel biomechanical insights. Spine J 2020;20:170516. https://doi.org/10.1016/j.spinee.2020.05.541.

[28] Kosaka H, Sairyo K, Biyani A, Leaman D, Yeasting R, Higashino K, et al. Pathomechanism of loss of elasticity and hypertrophy of lumbar ligamentum flavum in elderly patients with lumbar spinal canal stenosis. Spine (Phila Pa 1976) 2007;32:2805-11. https://doi.org/ 10.1097/BRS.0b013e31815b650f.

[29] Fujiwara A, Tamai K, An HS, Shimizu K, Yoshida H, Saotome K. The interspinous ligament of the lumbar spine: magnetic resonance images and their clinical significance. Spine (Phila Pa 1976) 2000;25:358-63. https://doi.org/10.1097/00007632-200002010-00017.

[30] Cheung PWH, Tam V, Leung VYL, Samartzis D, Cheung KMC, Luk $\mathrm{KDK}$, et al. The paradoxical relationship between ligamentum flavum hypertrophy and developmental lumbar spinal stenosis. Scoliosis Spinal Disord 2016;11:5-11. https://doi.org/10.1186/s13013-016-0088-5.
[31] Cornaz F, Widmer J, Farshad-Amacker NA, Spirig JM, Snedeker JG, Farshad M. Biomechanical contributions of spinal structures with different degrees of disc degeneration. Spine (Phila Pa 1976) 2020:1. Publish Ah. https://doi.org/10.1097/brs.0000000000003883.

[32] Seligman JV, Gertzbein SD, Tile M, Kapasouri A. Computer analysis of spinal segment motion in degenerative disc disease with and without axial loading. Spine (Phila Pa 1976) 1984;9:566-73. https://doi. org/10.1097/00007632-198409000-00006.

[33] Widmer J, Fornaciari P, Senteler M, Roth T, Snedeker JG, Farshad $M$. Kinematics of the spine under healthy and degenerative conditions: a systematic review. Ann Biomed Eng 2019. https://doi.org/ 10.1007/s10439-019-02252-x.

[34] Woo SLY, Orlando CA, Camp JF, Akeson WH. Effects of postmortem storage by freezing on ligament tensile behavior. J Biomech 1986;19:399-404. https://doi.org/10.1016/0021-9290(86)90016-3. 